\title{
Conjugated polymers as robust carriers for controlled delivery of anti-inflammatory drugs
}

\author{
Katarzyna Krukiewicz $\cdot$ Jerzy K. Zak
}

Received: 21 February 2014/Accepted: 29 April 2014/Published online: 21 May 2014

(C) The Author(s) 2014. This article is published with open access at Springerlink.com

\begin{abstract}
Conjugated polymers due to their reversible transition between the redox states are potentially able to immobilise and release ionic species. In this study, we have successfully developed a conducting polymer system based on poly(3,4-ethylenedioxythiophene) (PEDOT) for electrically triggered, local delivery of an ionic form of ibuprofen (IBU), a non-steroidal anti-inflammatory, and analgesic drug. It was shown that by changing the electropolymerisation conditions, the polymer matrix of specified IBU content can be synthesised. The electrochemical synthesis has been optimised to obtain the conducting matrix with the highest possible drug content. The process of electrically stimulated drug release has been extensively studied in terms of the dynamics of the controlled IBU release under varying conditions. The maximum concentration of the released IBU, $0.66( \pm 0.10) \mathrm{mM}$, was observed at the applied potential $E=-0.5 \mathrm{~V}$ (vs. Ag/ $\mathrm{AgCl})$. It was demonstrated that the immobilisation-release procedure can be repeated several times making the PEDOT matrix promising materials for controlled drug release systems applied e.g. in neuroprosthetics.
\end{abstract}

\section{Introduction}

Conjugated polymers are widely known for their electrical properties and have found numerous applications in areas such as organic solar cells, organic light-emitting diodes, supercapacitors, actuators, etc. [1-4]. Nowadays, conducting polymers have appeared also as materials suitable for biomedical engineering. For the last decade, numerous

K. Krukiewicz · J. K. Zak $(\bowtie)$

Silesian University of Technology, Gliwice, Poland

e-mail: jerzy.zak@polsl.pl biomedical applications based on biocompatible conducting polymers have been investigated, i.e. biosensors, molecular scaffolds, coatings for neuroprosthetics, and drug delivery systems [5-8]. Conjugated polymers, especially polypyrrole (PPy) and poly(3,4-ethylenedioxythiophene) (PEDOT), are now materials that are in a centre of attention for biomedical engineering; they have been proven to be biocompatible and may be introduced into a human body without any harmful effect on health [9-14]. Besides, their ability to conduct electricity is a significant advantage that enables efficient ways of processing and opens new potentially commercial applications, e.g. in neuroprosthetics [15-17]. It is known that implantable neural electrodes made of metal cause allergic reactions and inflammation, moreover, the introduction of implants carries the risk of infections [18]. Due to their biocompatibility, PPy and PEDOT films may act as an interface between the metal surface of implant and neural tissue reducing the risk of inflammation and glial scar formation [19-21].

The immobilisation of biologically active molecules in conjugated polymer matrices may lead to further increase in polymer biocompatibility $[20,22]$. Through an intentional choice of immobilised biomolecule, it is possible to prevent inflammation (immobilisation of anti-inflammatory drugs), infection (immobilisation of antibiotics), or brain oedema (immobilisation of steroid drugs) [23-25]. The immobilisation of neurotransmitters such as glutamate may be a step toward the development of retinal prosthesis [26]. The ion-exchangeable properties of intrinsically conducting polymers make them promising materials for controlled drug delivery systems. Biologically active compounds, which typically include ionic bonds in their structure, can be therefore immobilised in a conducting polymer matrix and released in a controlled way when stimulated electrically $[23,24]$. 
Numerous procedures of electrochemical immobilisation followed by electrically triggered drug release have been already presented in the literature. Piro et al. [27] demonstrated the electrochemical method of incorporation of oligonucleotides into PEDOT films followed by their release in a simple ion exchange process. Wadhwa et al. [18] developed PPy matrix for electrically controlled and local delivery of dexamethasone-a synthetic glucocorticoid anti-inflammatory drug. Esrafilzadeh et al. [28] presented conducting polymer fibres loaded with ciprofloxacin - an antibiotic that can be released or sustained in response to electrical stimulation. None of them, however, studied the stability of polymer matrix during stimulated drug release. This is an important question when considering medical applications of such matrices and their implementation into a human body [29-32].

In this study, we have described the synthesis of the conducting polymer matrix based on PEDOT as an example of biocompatible, conjugated polymer that recently has gained much interest in biomedical engineering [33-36]. The superior chemical and electrochemical stability is the main advantage of PEDOT compared to other currently applied conjugated polymers [37]. As a drug of interest, $\alpha$-methyl-4-(isobutyl)phenylacetic acid (ibuprofen, IBU) has been chosen, which is known as a non-steroidal anti-inflammatory and analgesic drug showing an ability to enhance wound healing [38-40]. The electrochemical immobilisation of IBU in PEDOT matrix has been investigated together with the study of electrically triggered drug release and the examination of the stability of polymer matrix against applied potential.

\section{Materials and methods}

\section{Materials}

3,4-Ethylenedioxythiophene (EDOT), lithium perchlorate of A.C.S. grade and IBU sodium salt (sodium $\alpha$-methyl-4(isobutyl)phenylacetate, IBU, $\mathrm{MW}=228.26 \mathrm{~g} \mathrm{~mol}^{-1}$ ) were obtained from Sigma Aldrich. Potassium chloride, dipotassium phosphate and monopotassium phosphate were obtained from POCh. All aqueous solutions were prepared with the use of deionised water.

\section{Instrumentation}

Electrochemical measurements and conditioning were performed in a three-electrode cell by use of $\mathrm{CH}$ Instruments 620 electrochemical workstation. The electrochemical synthesis of polymers was performed by means of cyclic voltammetry; the process of conditioning was carried out potentiostatically. The concentration of released
IBU was monitored by means of Hewlett Packard 8453 UV-Vis Diode Array Spectrophotometer.

\section{One-step synthesis of PEDOT/LiClO $/ 4$ IBU}

A three-electrode electrochemical cell was set up in a 3-ml electrochemical glass cell with $1 \mathrm{~cm}^{2}$ platinum foil working electrode, $\mathrm{Ag} / \mathrm{AgCl}$ as a reference electrode and glassy carbon rod as an auxiliary electrode. The polymer films containing IBU (PEDOT/ $\left./ \mathrm{LiClO}_{4} / \mathrm{IBU}\right)$ were obtained by means of cyclic voltammetry (CV) in aqueous solution containing $10 \mathrm{mM}$ EDOT, $10 \mathrm{mM}$ IBU, $0.1 \mathrm{M} \mathrm{LiClO}_{4}$, which was sonicated prior to the experiment for $30 \mathrm{~min}$. Typical polymer film was formed on Pt electrode within the potential range of $0-1.1 \mathrm{~V}(\mathrm{vs} . \mathrm{Ag} / \mathrm{AgCl})$ at the scan rate $v=100 \mathrm{mV} \mathrm{s}^{-1}$ for 25 potential cycles.

\section{Three-step synthesis of PEDOT/LiClO $4 / \mathrm{IBU}$}

In the three-step synthesis, the same three-electrode electrochemical cell was used as that described in the previous section. In the first step, the polymer film was obtained from aqueous solution containing $10 \mathrm{mM}$ EDOT and $0.1 \mathrm{M} \mathrm{LiClO}_{4}$ in the similar procedure employing cyclic voltammetry with a narrower potential range of $0-1.0 \mathrm{~V}$ (vs. $\mathrm{Ag} / \mathrm{AgCl}$ ), scan rate $v=100 \mathrm{mV} \mathrm{s}^{-1}$, and number of potential cycles equal to 50 .

In the second step, the original $\mathrm{ClO}_{4}{ }^{-}$dopant was removed from the polymer matrix by conditioning it at a reduction potential $E_{\text {red }}=-0.7 \mathrm{~V}$ (vs. $\mathrm{Ag} / \mathrm{AgCl}$ ) for

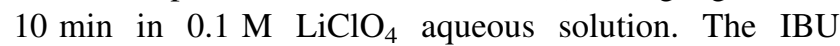
immobilisation occurred in the third step, when the polymer matrix after rinsing with deionised water was oxidised at a potential $E_{\mathrm{ox}}=0.8 \mathrm{~V}$ (vs. $\mathrm{Ag} / \mathrm{AgCl}$ ) in $0.4 \mathrm{M} \mathrm{IBU}$ aqueous solution for $10 \mathrm{~min}$.

\section{Release of IBU from PEDOT/LiClO $/ 4$ IBU matrix}

The concentration of IBU released from PEDOT/LiClO ${ }_{4} /$ IBU matrix was determined using UV-Vis spectroscopic measurements under potentiostatic conditions. The threeelectrode electrochemical cell was set up in a 2-mm quartz cuvette, in which the PEDOT/LiClO$/ / \mathrm{IBU}$-modified platinum-working electrode was mounted together with $\mathrm{Ag}$ wire used as a pseudoreference electrode, and graphite rod as an auxiliary electrode. Before measurements, the electrode covered with $\mathrm{PEDOT} / \mathrm{LiClO}_{4} / \mathrm{IBU}$ was soaked in PBS solution for 10 min to wash off any loosely attached IBU and unreacted monomer. In order to release IBU from PEDOT/ $/ \mathrm{LiClO}_{4} / \mathrm{IBU}$ matrix, a constant potential in the range -0.8 to $0.8 \mathrm{~V}$ (vs. $\mathrm{Ag} / \mathrm{AgCl}$ ) was stepped for a specified period of time. After each period of time at the given potential, the UV-Vis spectra were recorded for IBU 
released to the solution. All these measurements of the controlled release of the immobilised IBU were performed in the $\mathrm{pH} 6.5$ phosphate buffer solution, $\mathrm{PBS}$, containing $0.15 \mathrm{M} \mathrm{KCl}, 0.006 \mathrm{M} \mathrm{K}_{2} \mathrm{HPO}_{4}, 0.001 \mathrm{M} \mathrm{KH}_{2} \mathrm{PO}_{4}$.

Stability of PEDOT/LiClO $4 / \mathrm{IBU}$ matrix at variable potential

The stability of PEDOT/LiClO $4 / \mathrm{IBU}$ matrix exposed to a given electrode potential was determined in terms of the charge storage capacity, CSC, of the matrix. That factor was calculated as the electric charge integrated under corresponding $\mathrm{CV}$ curve during one $\mathrm{CV}$ cycle

$\mathrm{CSC}=\int_{t_{1}}^{t_{2}} I(t) \mathrm{d} t$

where $t_{1}$ is the beginning of $\mathrm{CV}$ cycle, $t_{2}$ is the end of $\mathrm{CV}$ cycle.

The CVs were recorded for the modified platinum electrode in PBS after $20 \mathrm{~min}$ exposition to a given potential.

\section{Results and discussion}

One-step synthesis of PEDOT/LiClO 4 /IBU

As many other anions, IBU in its ionic form (Scheme 1) is able to participate in the polymer doping process, which is a part of the electropolymerisation reaction. Molecules like EDOT when oxidised (chemically or electrochemically) extend their conjugated bonding system, forming positively charged polymer chain that is stabilised by doping of anions from the reaction environment. Under the electrochemical conditions, the polymerisation/doping process occurs at positive potentials applied to the electrode in the electrolyte solution containing monomer. Thus, a presence of $\mathrm{IBU}^{-}$in the electrolyte solution may lead to its immobilisation in the polymer matrix, which is created on the electrode surface. This process is shown in Scheme 2.

The current versus potential curves recorded in subsequent scans make it possible to observe the progress of electropolymerisation process, as shown in Fig. 1. Two sets of CV curves were collected, the first for the solution containing EDOT, $\mathrm{LiClO}_{4}$ and IBU (Fig. 1a) and the second for the case, when IBU was not present in the solution (Fig. 1b). In both cases, the

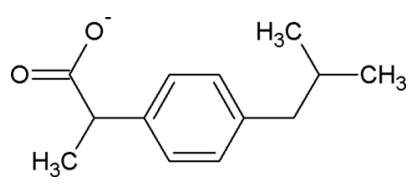

Scheme 1 The chemical structure of anionic form of IBU increase of current indicates that the monomer is irreversibly oxidised near $1.1 \mathrm{~V}$ (vs. $\mathrm{Ag} / \mathrm{AgCl}$ ). Also for both data sets, the gradually increasing currents confirm formation of conductive deposits, typical for the electropolymerisation process. At the same number of potential scans, the final level of the resulting currents and corresponding electric charge are evidently different, indicating a slower process of electrochemical polymerisation of EDOT in case of IBU presence in solution

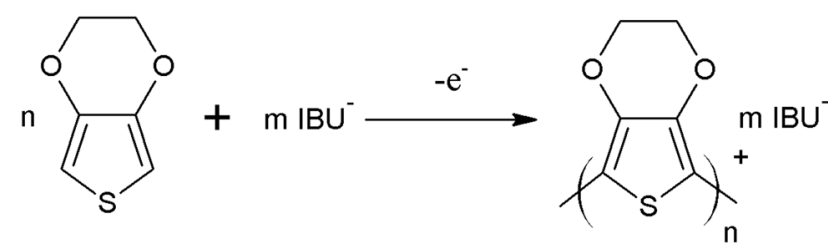

Scheme 2 The schematic representation of the immobilisation of IBU into PEDOT matrix during the process of electropolymerisation
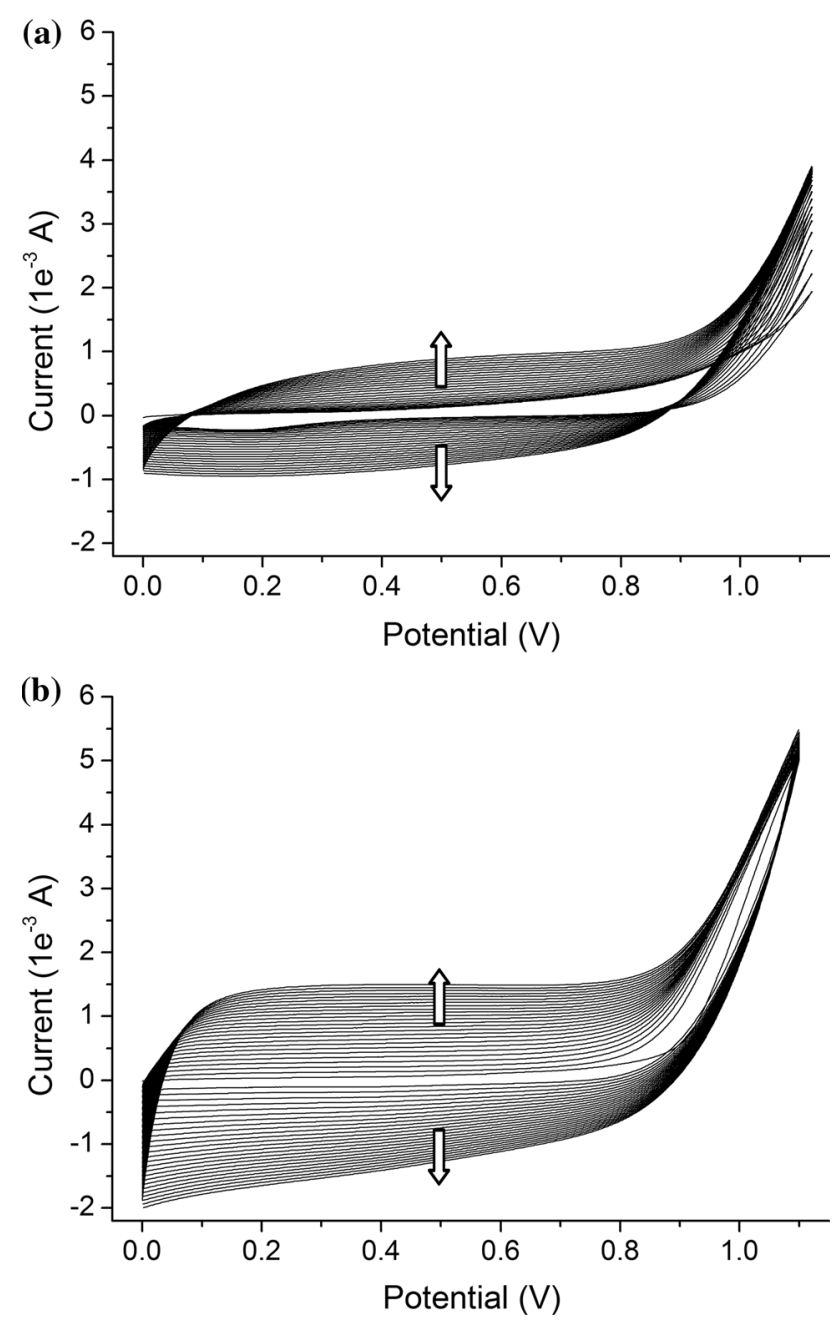

Fig. 1 The CV curves recorded during electrochemical polymerisation of $10 \mathrm{mM}$ EDOT in $0.1 \mathrm{M} \mathrm{LiClO}_{4}$ aqueous solution in the presence of $10 \mathrm{mM} \mathrm{IBU}(\mathbf{a})$, and without IBU (b); potential range $0-1.1 \mathrm{~V}$ (vs. $\mathrm{Ag} / \mathrm{AgCl}$ ), scan rate $v=100 \mathrm{mV} / \mathrm{s}, 25$ potential cycles 


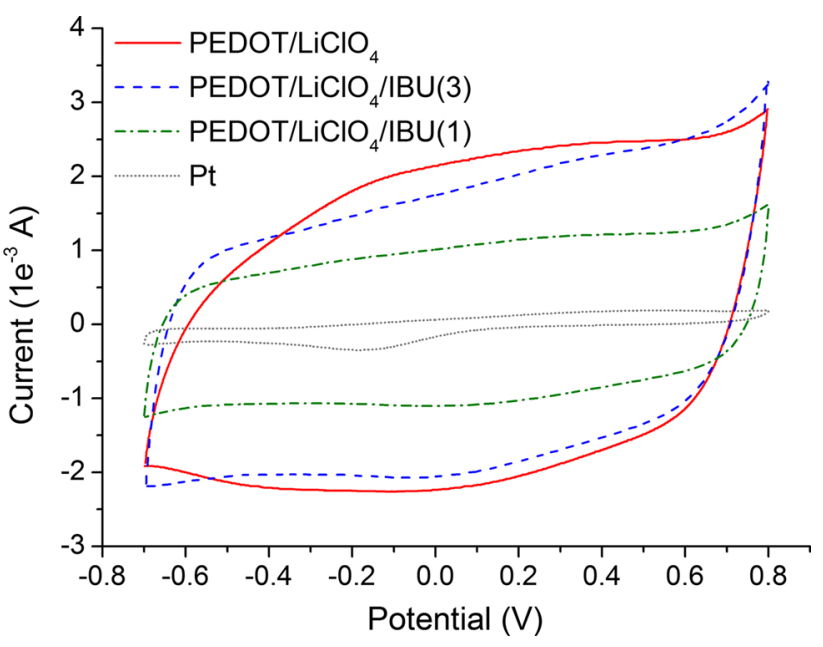

Fig. 2 The CVs recorded on PEDOT/LiClO 4 (red line) and PEDOT/ $\mathrm{LiClO}_{4} / \mathrm{IBU}$ working electrodes synthesised in one-step (1) (green line and dots) and three-step(3) (blue dotted line) procedure, and $\mathrm{CV}$ recorded on bare Pt-working electrode in $0.1 \mathrm{M} \mathrm{LiClO}_{4}$ solution (grey dots); scan rate $\mathrm{v}=100 \mathrm{mV} / \mathrm{s}$

(Fig. 1a). This may be due to the fact that the molecule of IBU is significantly larger than the molecule of electrolyte, thus the incorporation of drug into the structure of the polymer is less spatially favourable. The preliminary results indicated that the molar ratio of EDOT to IBU equal to 1:1 produces a polymer with the highest drug content, approximately $60 \mu \mathrm{g} \mathrm{cm}^{-2}$ (mass of IBU immobilised in the unit surface area of Pt electrode). The charge storage capacity, CSC, calculated for PEDOT/LiClO $4 / \mathrm{IBU}(22.3 \mathrm{mC})$ was however not as large as for PEDOT/ $/ \mathrm{LiClO}_{4}$ film $(43.6 \mathrm{mC}$ ) polymerised under analogous conditions as indicate the CV curves shown in Fig. 2. The CSC factor used in here is simply current integrated along the potential axis recorded for the polymer-coated electrode placed in the pure electrolyte solution. It was found that the use of higher IBU concentrations suppresses the formation of polymer film, therefore it has adverse effects on its charge storage capacity. When IBU was present at lower concentration in the monomer solution $(1,2$, or $5 \mathrm{mM})$, the amount of IBU immobilised in the polymer film was as low as 5, 6 and $11 \mu \mathrm{g} \mathrm{cm}^{-2}$, respectively.

\section{Three-step synthesis of PEDOT/LiClO $4 / \mathrm{IBU}$}

The three-step synthesis of $\mathrm{PEDOT} / \mathrm{LiClO}_{4} / \mathrm{IBU}$ matrix allows to separate the processes of electropolymerisation and immobilisation. In this procedure, the polymer film is formed in pure electrolyte solution, thus it is doped only with perchlorate ions. Electrochemical reduction of the film eliminates to some extent that dopant from the polymer. Oxidation of the polymer is then carried out in the aqueous solution of IBU only. The $\mathrm{CVs}$ recorded on $\mathrm{Pt}$ electrodes modified with $\mathrm{PEDOT} / \mathrm{LiClO}_{4}$ and PEDOT/
$\mathrm{LiClO}_{4} / \mathrm{IBU}$ obtained via one-step (1) and three-step (3) procedures are compared in Fig. 2. The average current magnitude of $\mathrm{PEDOT} / \mathrm{LiClO}_{4} / \mathrm{IBU}(3)$ is larger than for PEDOT/LiClO $4 / \mathrm{IBU}(1)$ and only slightly smaller than that for $\mathrm{PEDOT} / \mathrm{LiClO}_{4}$. Hence, it may be concluded that a three-step synthesis route results in a matrix with a higher CSC $(40.9 \mathrm{mC})$ than matrix synthesised according to the one-step procedure $(22.3 \mathrm{mC})$, only slightly smaller than CSC of $\mathrm{PEDOT} / \mathrm{LiClO}_{4}$ film $(43.6 \mathrm{mC})$.

\section{Release of IBU from PEDOT/LiClO $4 / \mathrm{IBU}$}

During the synthesis, anionic compounds are immobilised in a positively charged conducting polymer matrix to maintain a neutral charge of the polymer backbone. When the sufficiently negative voltage is applied to the matrix, the anionic dopant becomes unnecessary, therefore it is released to the solution. The schematic representation of the electrically triggered drug release from conducting polymer matrix is shown in Scheme 3.

The process of IBU release from $\mathrm{PEDOT} / \mathrm{LiClO}_{4} / \mathrm{IBU}$ matrix was monitored under potentiostatic conditions using UV-Vis spectroscopy. Calibration curve was plotted for absorbance versus IBU concentration (where $y$ is the absorbance and $x$ is the IBU concentration in $\mathrm{mM}$ ). A linear relationship was observed between 0.01 and $1 \mathrm{mM}$ IBU concentration satisfying the equation $y=0.0176 x+1.9365$ $\left(R^{2}=0.9992\right)$.

In order to release IBU from $\mathrm{PEDOT} / \mathrm{LiClO}_{4} / \mathrm{IBU}$ matrix, a constant potential in the range between -0.8 and $0.8 \mathrm{~V}$ (vs. $\mathrm{Ag} / \mathrm{AgCl}$ ) was applied to the matrix placed in the cuvette. The absorbance at $\lambda=222 \mathrm{~nm}$, which is characteristic for IBU, was recorded versus time at a given potential. The changes of absorbance spectra in time for the matrix stimulated with $E=-0.5 \mathrm{~V}$ (vs. $\mathrm{Ag} / \mathrm{AgCl}$ ) are presented in Fig. 3.

The time profiles of IBU release from the matrix stimulated with $E=-0.5 \mathrm{~V}$ (vs. $\mathrm{Ag} / \mathrm{AgCl}$ ) and matrix maintained under open circuit conditions are presented in Fig. 4. As it can be seen, for the non-stimulated matrix (open circuit potential), IBU was released in small concentration, six times lower than for the matrix stimulated with the reducing potential $E_{\text {red }}=-0.5 \mathrm{~V}$ (vs. $\mathrm{Ag} / \mathrm{AgCl}$ ). This result evidently confirms the effect of the applied potential

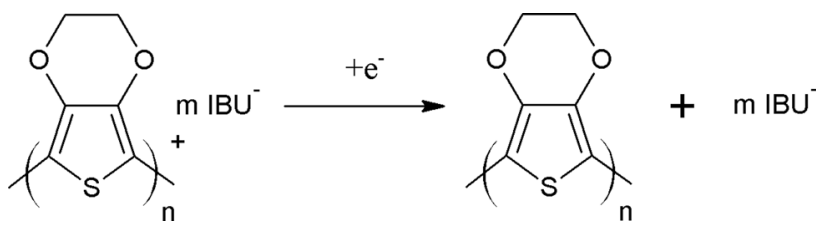

Scheme 3 The schematic representation of electrically triggered drug release from $\mathrm{PEDOT} / \mathrm{LiClO}_{4} / \mathrm{IBU}$ matrix 


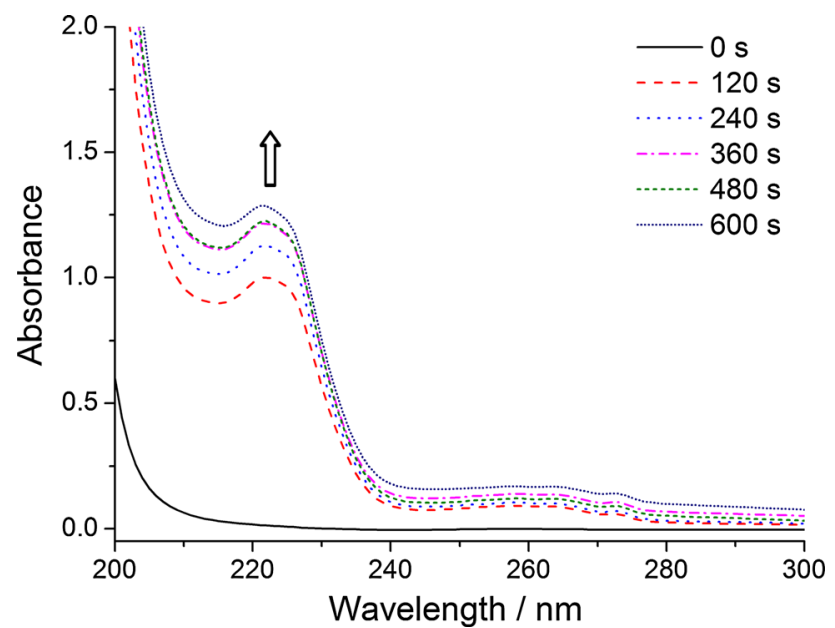

Fig. 3 The change of absorbance spectra in time for IBU released from the matrix stimulated with $E=-0.5 \mathrm{~V}$ (vs. $\mathrm{Ag} / \mathrm{AgCl}$ )

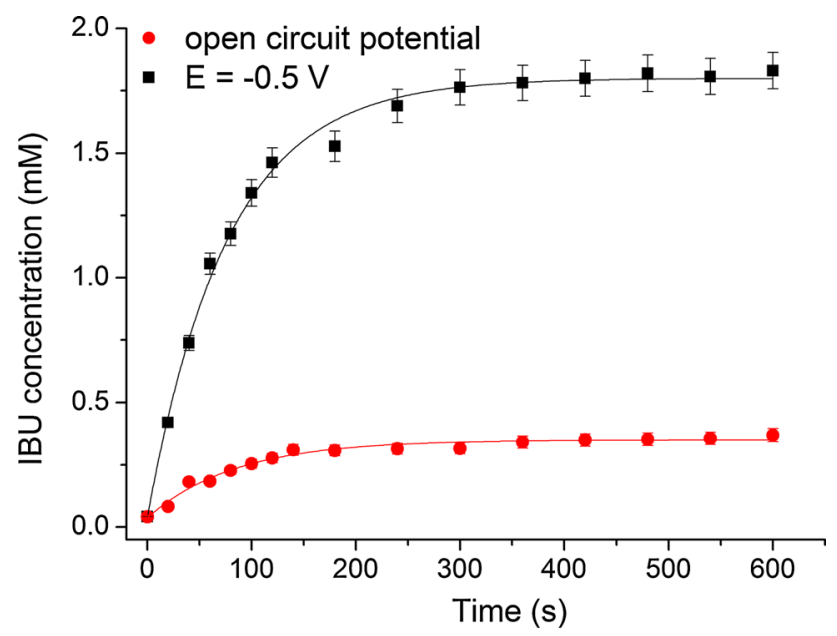

Fig. 4 The concentration of the released IBU calculated from UVVis data as a function of time of the electric stimulation of PEDOT/ $\mathrm{LiClO}_{4} / \mathrm{IBU}$ matrices with potential $E=-0.5 \mathrm{~V}$ (black square) and under open circuit conditions (red circle). The plain line is only a visual guide

on the process of the drug release. The greatest quantity of IBU appears to be released during the first $2 \mathrm{~min}$ of the electric stimulation, as compared to longer times. It suggests that only a small portion of IBU molecules is imbedded deeply within the polymeric structure of the matrix; also the process of full reduction of PEDOT is practically completed within the applied stimulation time. Under the described conditions, a total time below $10 \mathrm{~min}$ is required to reach equilibrium $\left(t_{\text {equil }}\right)$ in the system, then the solution concentration of IBU is stable.

Since the ratio of the amounts of oxidised and reduced forms of the conjugated polymer is strictly determined by the potential applied to the polymer film, therefore one can

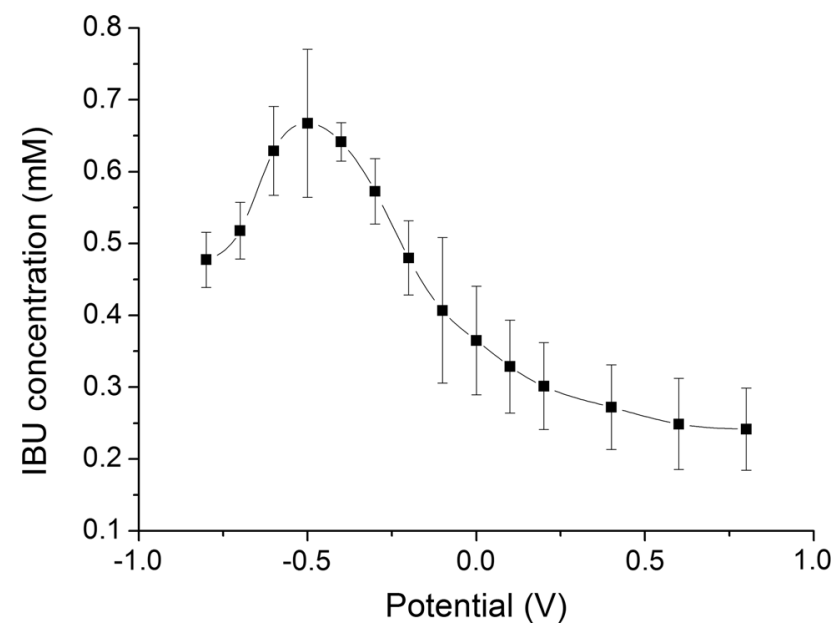

Fig. 5 The concentration of IBU released as a function of the potential applied to $\mathrm{PEDOT} / \mathrm{LiClO}_{4} / \mathrm{IBU}$ matrix; the plain line is only a visual guide. The error bars represent standard deviations for five independent measurements

expect variation in the concentration of IBU released to the solution as a function of the value of stimulating potential. These data were collected for $t_{\text {equil }}=10 \mathrm{~min}$ for polymer films of approximately the same amount of immobilised IBU. The resulting concentration of IBU released as a function of applied potential is shown in Fig. 5. The maximum concentration of IBU released, $0.66( \pm 0.10)$ $\mathrm{mM}$, was observed when the potential $E=-0.5 \mathrm{~V}$ (vs. $\mathrm{Ag} / \mathrm{AgCl}$ ) was applied. When PEDOT/ $/ \mathrm{LiClO}_{4} / \mathrm{IBU}$ was subjected to more negative potentials, the concentration of released IBU decreased. This is caused by the effect of n-doping of negatively charged polymer matrix with cations present in PBS solution: the incorporation of potassium cations occurs instead of the release of the anionic form of IBU. The concentration of IBU released decreases as the value of applied potential increases since the positively charged film holds the anionic drug; the concentration of IBU released at $E=0.8 \mathrm{~V}$ (vs. $\mathrm{Ag} / \mathrm{AgCl}$ ) was 0.21 $( \pm 0.05) \mathrm{mM}$, which is one-third of the concentration released at $E=-0.5 \mathrm{~V}$ (vs. $\mathrm{Ag} / \mathrm{AgCl}$ ). These results are consistent with the mentioned above effects of the applied potential on the redox state of conjugated polymer. The application of negative potentials results in facilitated release of anionic co-dopant, IBU, while the application of positive potential results in the drug retention.

The capacity of the polymer matrix towards the amount of immobilised IBU generally depends on the amount of polymer; the last in turn is easy to control in the process of electropolymerisation by variation of the number of $\mathrm{CV}$ cycles. The relation between the drug capacity of the matrix and the conditions of electrochemical polymerisation, in terms of number of $\mathrm{CV}$ cycles, is presented in Fig. 6 . The data indicate nearly linear relation between the 


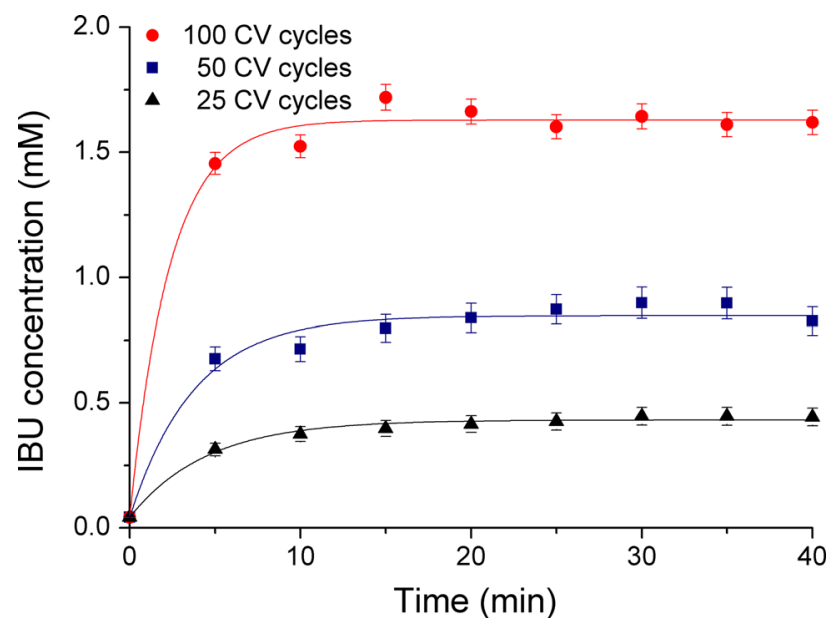

Fig. 6 The concentration of IBU released versus stimulation time of polymer matrix for different film thicknesses measured as electropolymerisation number of $\mathrm{CV}$ cycles: $25 \mathrm{CV}$ cycles (black triangle), $50 \mathrm{CV}$ cycles (blue square), $100 \mathrm{CV}$ cycles (red circle). PEDOT/ $\mathrm{LiClO}_{4} / \mathrm{IBU}$ matrices were electrically stimulated at potential $E=$ $-0.5 \mathrm{~V}$ (vs. $\mathrm{Ag} / \mathrm{AgCl}$ ). The plain line is only a visual guide

electropolymerisation time and the drug capacity; the first is represented by the number of CV scans, whereas the other is expressed in terms of the concentration of released IBU. These results demonstrate that it is possible to synthesise PEDOT/LiClO $/$ /IBU matrix of a specified IBU content that can be further effectively released.

Since the three-step synthesis of PEDOT/LiClO $4 / \mathrm{IBU}$ allows to separate the processes of electropolymerisation and immobilisation, it should be possible to apply the immobilisation-release procedure several times for the same matrix. Again, the curves representing the concentration of IBU released versus time were plotted for the PEDOT/LiClO $4 / \mathrm{IBU}$ matrix that was subjected to four consecutive immobilisation-release cycles. The plot of the concentration of released IBU calculated from the corresponding UV-Vis data is shown in Fig. 7. After the first cycle, the concentration of released IBU was equal to 0.70 $( \pm 0.05) \mathrm{mM}$, whereas each subsequent immobilisation/ release cycle appeared to be less efficient; in the forth cycle the concentration of IBU released was reduced to 0.34 $( \pm 0.02) \mathrm{mM}$. The observed effect is caused by the gradual degradation of $\mathrm{PEDOT} / \mathrm{LiClO}_{4} / \mathrm{IBU}$ matrix that usually occurs when the polymer is exposed to oxidative potential exceeding its optimal value (overoxidation). If, however, the potential is carefully chosen, the polymer degradation during its destructive oxidation can be optimised. On the other side, the matrix exposed to negative potentials for longer times (or at no potential applied) keeps its CSC factor practically unchanged.

The gradual loss of the matrix loading effectiveness, as observed in the spectral measurements, is likely caused by

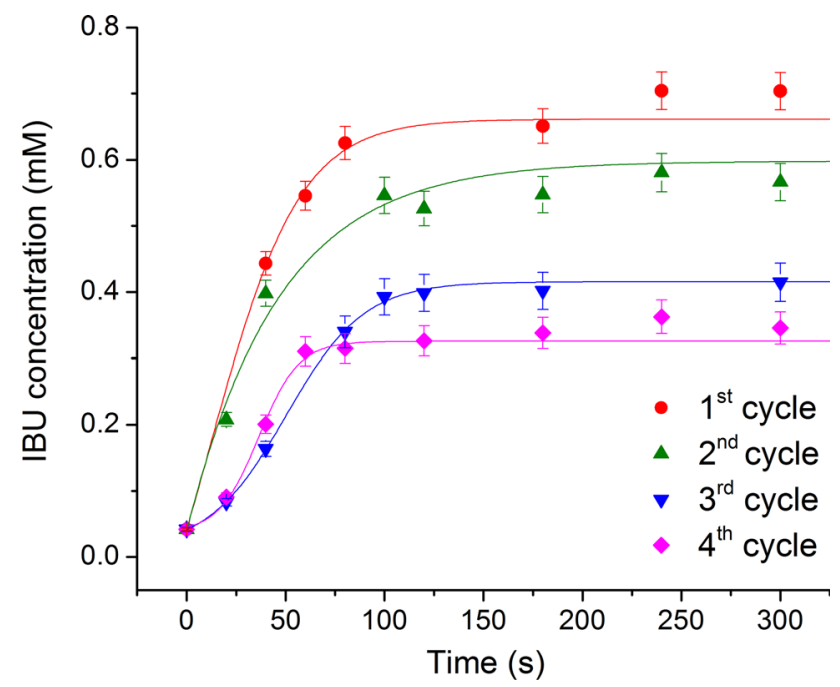

Fig. 7 The concentration of IBU released versus time for PEDOT/ $\mathrm{LiClO}_{4} / \mathrm{IBU}$ matrix subjected to four immobilisation-release cycles: first cycle (red circle), second cycle (green triangle), third cycle (blue inverted triangle), forth cycle (pink diamond). The plain line is only a visual guide

Table 1 The charge storage capacity (CSC) of PEDOT/LiClO ${ }_{4} / \mathrm{IBU}$ matrix calculated from $\mathrm{CV}$ data before and after the first, second, third and forth immobilisation-release cycle as compared to CSC of bare $\mathrm{Pt}$ electrode

\begin{tabular}{lc}
\hline Type of surface & CSC (mC) \\
\hline Bare Pt & 3.77 \\
Modified Pt before 1st load/release cycle & 53.26 \\
Modified Pt after 1st load/release cycle & 39.48 \\
Modified Pt after 2nd load/release cycle & 25.00 \\
Modified Pt after 3rd load/release cycle & 15.53 \\
Modified Pt after 4th load/release cycle & 8.93 \\
\hline
\end{tabular}

a degradation of the polymer film. The $\mathrm{CV}$ curves recorded after each IBU load/release cycle reveal decreasing electric charge. These data shown in terms of CSC values (Table 1) are consistent with the spectral results, which all together give a proof of a limited durability of the PEDOT matrix when multiple IBU- loadings are attempted.

\section{Conclusions}

In this study, a PEDOT-based conducting polymer system for electrically triggered delivery of an ionic form of IBU has been developed. The most favourable electropolymerisation conditions for achieving good electrochemical properties along with the highest drug content have been found and the process of electrically stimulated drug release has been extensively studied. The time profiles have provided the 
information about the dynamics of drug release, while application of different potentials has shown that the process of the drug release may be effectively controlled. The concentration of the released IBU has been shown to be dependent on the redox state of the polymer matrix, therefore application of negative potential resulted in the drug release, while the positive potentials resulted in the drug retention.

The proposed three-step synthesis of PEDOT/LiClO 4 IBU matrix allowed the separation of the electropolymerisation and immobilisation processes, which provided the matrix of a superior charge storage capacity. It was demonstrated that the immobilisation-release procedure can be repeated several times making the drug delivery system able to be reloaded. However, decreasing electric charge and gradual loss of the matrix loading efficiency after each IBU load/release cycle show the limited durability of the PEDOT matrix when multiple IBU-loadings are attempted. That is why further research should be focused on the optimisation of the loading-reloading conditions, especially on the careful choice of the oxidation and reduction potentials. If, however, the aforementioned conditions are optimised and the durability of PEDOT is improved, the $\mathrm{PEDOT} / \mathrm{LiClO}_{4} / \mathrm{IBU}$ system is expected to find application as a novel carrier for controlled-release local delivery of anti-inflammatory drug for biomedical engineering.

Acknowledgements This work was supported by the Polish National Science Centre (Preludium 2012/07/N/ST5/01878).

Open Access This article is distributed under the terms of the Creative Commons Attribution License which permits any use, distribution, and reproduction in any medium, provided the original author(s) and the source are credited.

\section{References}

1. Kim WH, Mäkinen AJ, Nikolov N et al (2002) Molecular organic light-emitting diodes using highly conducting polymers as anodes. Appl Phys Lett 80:3844-3846

2. Matoetoe MC (2010) A review of dye incorporated conducting polymers application as sensors and in solar cells. Mater Sci Forum 657:208-230

3. Mastragostino M, Arbizzani C, Soavi F (2002) Conducting polymers as electrode materials in supercapacitors. Solid State Ionics 148:493-498

4. Coskun Y, Cirpan A, Toppare L (2007) Construction of electrochromic devices using thiophene based conducting polymers. J Mater Sci 42:368-372. doi:10.1007/s10853-006-1076-6

5. Guimard NK, Gomez N, Schmidt CE (2007) Conducting polymers in biomedical engineering. Prog Polym Sci 32:876-921

6. Ravichandran R, Sundarrajan S, Venugopal JR et al (2010) Applications of conducting polymers and their issues in biomedical engineering. J R Soc Interface 7:559-579

7. Svennersten K, Larsson KC, Berggren M et al (2011) Organic bioelectronics in nanomedicine. Biochim Biophys Acta 1810: 276-285
8. Subramanian A, Krishnan UM, Sethuraman S (2009) Development of biomaterial scaffold for nerve tissue engineering: biomaterial mediated neural regeneration. J Biomed Sci 16:108-119

9. Geetha S, Rao CRK, Vijayan M et al (2006) Biosensing and drug delivery by polypyrrole. Anal Chim Acta 568:119-125

10. George PM, Lyckman AW, LaVan DA et al (2005) Fabrication and biocompatibility of polypyrrole implants suitable for neural prosthetics. Biomaterials 26:3511-3519

11. Asplund M, Thaning E, Lundberg J et al (2009) Toxicity evaluation of PEDOT/biomolecular composites intended for neural communication electrodes. Biomed Mater 4:1-12

12. Bennet D, Kim S (2011) Implantable microdevice for peripheral nerve regeneration: materials and fabrications. J Mater Sci 46:4723-4740. doi:10.1007/s10853-011-5510-z

13. Ateh DD, Navsaria HA, Vadgama P (2009) Polypyrrole-based conducting polymers and interactions with biological tissues. J R Soc Interface 3:741-752

14. Kim DH, Richardson-Burns SM, Hendricks JL et al (2007) Effect of immobilised nerve growth factor on conductive polymers: electrical properties and cellular response. Adv Funct Mater 17:79-86

15. Asplund $M$, von Holst $H$, Inganäs $\mathrm{O}$ (2008) Composite biomolecule/PEDOT materials for neural electrodes. Biointerphases 3:83-93

16. Thompson BC, Richardson RT, Moulton SE et al (2010) Conducting polymers, dual neurotrophins and pulsed electrical stimulation-dramatic effects on neurite outgrowth. J Control Release 141:161-167

17. Abidian MR, Martin DC (2009) Multifunctional nanobiomaterials for neural interfaces. Adv Funct Mater 19:573-585

18. Wadhwa R, Lagenaur CF, Cui XT (2006) Electrochemically controlled release of dexamethasone from conducting polymer polypyrrole coated electrode. J Control Release 110:531-541

19. Gomez N, Schmidt CE (2007) Nerve growth factor-immobilised polypyrrole: bioactive electrically conducting polymer for enhanced neurite extension. J Biomed Mater Res A 81: 135-149

20. Green RA, Lovell NL, Poole-Warren LA (2009) Cell attachment functionality of bioactive conducting polymers for neural interfaces. Biomaterials 30:3637-3644

21. Cogan SF (2008) Neural stimulation and recording electrodes. Annu Rev Biomed Eng 10:275-309

22. Xiao YH, Li CM, Toh ML et al (2008) Adenosine 50-triphosphate incorporated poly(3,4-ethylenedioxythiophene) modified electrode: a bioactive platform with electroactivity, stability and biocompatibility. J Appl Electrochem 38:1735-1741

23. Svirskis D, Travas-Sejdic J, Rodgers A et al (2010) Electrochemically controlled drug delivery based on intrinsically conducting polymers. J Control Release 146:6-15

24. Sassolas A, Blum LJ, Leca-Bouvier BD (2012) Immobilisation strategies to develop enzymatic biosensors. Biotechnol Adv 30:489-511

25. Vela MH, de Jesus DS, Couto CMCM et al (2003) Electroimmobilisation of MAO into a polypyrrole film and its utilization for amperometric flow detection of antidepressant drugs. Electroanalysis $15: 133-138$

26. Paul N, Muller M, Paul A et al (2013) Molecularly imprinted conductive polymers for controlled trafficking of neurotransmitters at solid-liquid interfaces. Soft Matter 9:1364-1371

27. Piro B, Pham MC, Ledoan T (1999) Electrochemical method for entrapment of oligonucleotides in polymer-coated electrodes. J Biomed Mater Res A 46:566-572

28. Esrafilzadeh D, Razal JM, Moulton SE et al (2013) Multifunctional conducting fibres with electrically controlled release of ciprofloxacin. J Control Release 169:313-320 
29. Boretius T, Schuettler M, Stieglitz T (2011) On the stability of poly-ethylenedioxythiopene as coating material for active neural implants. J Artif Organs 35:245-248

30. Green RA, Lovell NH, Wallace GG et al (2008) Conducting polymers for neural interfaces: challenges in developing an effective long-term implant. Biomaterials 29:3393-3399

31. Thaning EM, Asplund MLM, Nyberg TA et al (2010) Stability of poly(3,4-ethylene dioxythiophene) materials intended for implants. J Biomed Mater Res B 93:407-415

32. Che J, Xiao Y, Zhu X et al (2008) Electro-synthesised PEDOT/ glutamate chemically modified electrode: a combination of electrical and biocompatible features. Polym Int 57:750-755

33. Rozlosnik N (2009) New directions in medical biosensors employing poly(3,4-ethylenedioxy thiophene) derivative-based electrodes. Anal Bioanal Chem 395:637-645

34. Santhosh P, Manesh KM, Uthayakumar S et al (2009) Fabrication of enzymatic glucose biosensor based on palladium nanoparticles dispersed onto poly(3,4-ethylenedioxythiophene) nanofibers. Bioelectrochemistry 75:61-66
35. Ho KC, Yeh WM, Tung TS et al (2005) Amperometric detection of morphine based on poly(3,4-ethylenedioxythiophene) immobilised molecularly imprinted polymer particles prepared by precipitation polymerisation. Anal Chim Acta 542:90-96

36. Nien PC, Chen PY, Hsu CY et al (2011) On-chip glucose biosensor based on enzyme entrapment with pre-reaction to lower interference in a flow injection system. Sens Actuators B 157:64-71

37. Kros A, van Hövell SWFM, Sommerdijk NAJM et al (2001) Poly(3,4-ethylenedioxythiophene)-based glucose biosensors. Adv Mater 13:1555-1557

38. Schreml S, Szeimies R, Prantl L et al (2009) Wound healing in the 21st century. J Am Acad Dermatol 63:866-879

39. Shao F, Liu L, Fan K, Cai Y, Yao J (2012) Ibuprofen loaded porous calcium phosphate nanospheres for skeletal drug delivery system. J Mater Sci 47:1054-1058. doi:10.1007/s10853-0115894-9

40. Tsatsanis C, Androulidaki A, Venihaki M et al (2006) Signalling networks regulating cyclooxygenase-2. Int J Biochem Cell Biol 38:1654-1661 